\title{
Steroid Myopathy: Understanding the Pathogenesis
}

\author{
Monica Canepari* \\ Department of Molecular Medicine and Interuniversity Institute of Myology, University of Pavia, Italy
}

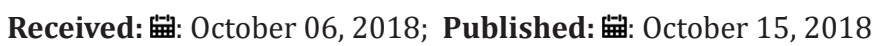

*Corresponding author: Monica Canepari, Department of Molecular Medicine and Interuniversity Institute of Myology University of Pavia, Italy

\begin{abstract}
Steroid myopathy is a consequence of glucocorticoid administration and excess occurring in Cushing's syndrome. It is characterized by muscle weakness, which impairs patients' capacity to perform everyday activities. The relevance of steroid myopathy is very high especially given the large number of patients suffering from inflammatory diseases that are treated with glucocorticoids. The major pathogenetic mechanisms hypothesized are: imbalance between protein synthesis and degradation, impairment of mitochondrial function and redox imbalance. The triggers of the latter phenomena and muscle damage are still unknown. The latter issues are still unsatisfactorily settled mainly because analyses are mostly focused at times when steroid myopathy had fully developed making it difficult to take apart the phenomena causing the myopathy from those being a consequence of it. To identify the triggering mechanisms involved in the pathogenesis of steroid myopathy the very early stages of the phenomenon must be investigated. This short review describes the effect of short-term corticosteroid administration on muscle functional, structural and molecular adaptations in humans and mice.
\end{abstract}

Keywords: Steroid Myopathy; Glucocorticoids; Muscle Atrophy; Dexamethasone

Abbreviations: Akt: Protein Kinase B; CSA: Cross-Sectional Area; DEX: Dexamethasone; FoxO: Fork-Head Box 0; mTOR: Mammalian Target of Rapamycin; MuRF-1: Muscle-Specific Ring Finger Protein-1; NRF2: Nuclear Factor (Erythroid-Derived 2)-Like2 (Nre212); PGC-1 $\alpha$ : Peroxisome Proliferative Activated Receptor- $\Gamma$ Coactivator $1 \alpha$; Po/CSA: Specific Force; SD: Standard Deviation; Sol: Soleus Muscle; SOD1: Cu/Zn Superoxide Dismutase; UPS: Ubiquitin Proteasome System; VL: Vastus Lateralis Muscle

\section{Introduction}

This short review will focus on

a) Quantitative and qualitative adaptations of individual muscle fibers of healthy subjects to short-term glucocorticoid administration.

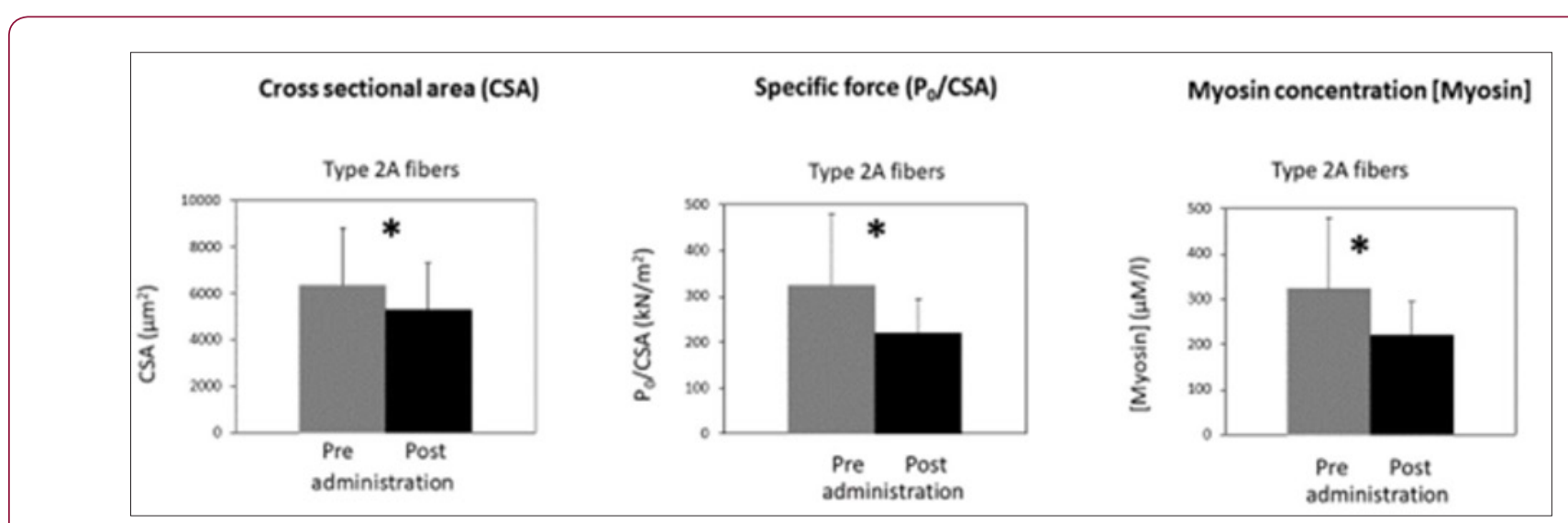

b) Molecular adaptations of isolated muscles from mice to a single glucocorticoid administration. The goal is to contribute to the understanding the pathogenesis of the steroid myopathy.

Figure 1. 
Quantitative and Qualitative Adaptations of Individual Muscle Fibers of Healthy Subjects to Short-Term Glucocorticoid Administration

The work of Minetto et al. [1] was the first in vivo study to describe the effects of a week dexamethasone (DEX) administration on skeletal muscle of healthy subjects that comprised both mechanical and molecular analysis. Five young men volunteered to participate in the study. Muscle samples were taken from the dominant vastus lateralis (VL) muscle by needle biopsy using the Bergstrom technique. The voluntaries were subjected to 7 days of oral dexamethasone (DEX) (8mg once daily) administration. Dexamethasone was specifically selected, because steroid myopathy has been reported to be associated with the use of fluorinated steroids [2,3]. Chemically skinned individual muscle fibers were dissected from biopsy samples and analyzed in terms of their cross-sectional area (CSA), contractile properties of specific force (Po/CSA) [4] and quantification of myosin concentration [5]. After a week of DEX administration, type 2A fibers (the most representative in VL muscle) underwent atrophy, decreased their specific force, and decreased their myosin concentration (Figure 1).

Although muscle atrophy is a well-known side effect of a longterm glucocorticoid excess, this study is the first showing that the short-term administration of glucocorticoids, in doses well within the range used clinically, reduced muscle fiber CSA and myosin concentration, this has been observed previously in young healthy subjects after immobilization [5] and in atrophic fibers of elderly subjects [6]. Because the intervention duration was short, and all subjects continued their normal daily activities during the administration, it is unlikely that the decrease in CSA and the myosin loss were due to muscle disuse. A reduction in circulating muscle proteins points toward the anti-anabolic effect of glucocorticoids as the most plausible explanation for the decrease in CSA and myosin loss. Myosin loss and a decrease in acto-myosin interaction could also explain the observed reduction in specific force of muscle fibers. A further mechanism that could account for the reduction in specific force is impairment of excitation-contraction coupling. However, as the experiments were done on chemically skinned muscle fibers lack of excitation contraction coupling, the latter cannot be involved in specific force loss, and myosin loss remains the most likely.

\section{Mechanism}

The obtained results suggest that the glucocorticoid-induced quantitative and qualitative adaptations of muscle fibers develops, even in healthy subjects, after only a few days of glucocorticoid administration.

\section{Molecular Adaptations of Isolated Muscles from Mice to A Single Glucocorticoid Administration}

It has been shown that the processes underlying steroid myopathy in humans develop just few days after glucocorticoid administration [1]. The next step was to establish a causal relationship between muscle structure and function alterations and specific molecular events. To address such issues, we studied steroid myopathy starting at very early times and through its developing in animals. C57BL/6 mice were subjected either to a "chronic" treatment or to an "acute" treatment by DEX. In the chronic treatment, mice were subjected to daily peritoneal injections of DEX for 3, 7 and 15 days and analyses were performed 24 hours after the last injection in order to assess the degree of muscle atrophy. In the acute treatment, mice were subjected to a single DEX injection and analyses of steady state adaptations in gene expression of key components of intracellular pathways controlling muscle mass and metabolism were performed 1, 3 and 10 hours after the injection.

The major intracellular signalling pathways controlling protein degradation (Foxo3a, Murf-1. Atrogin1), protein synthesis (AKT/ mTOR, and Myostatin) muscle metabolism (Pgc1 $\alpha$, citrate synthase) and redox balance (Nrf2, Sod1, and Catalase) in response to DEX treatment were studied in VL and soleus muscle (SOL). Results showed: (i) The presence of atrophy in both muscles after "chronic" DEX treatment (Figure 2) and (ii) An increased expression of MuRF-1, activator of the ubiquitin-proteasome protein degradation system (UPS) and Myostatin, an inhibitor of protein synthesis, in both muscles, 1 hour after a single injection (Figure 3). Results suggest that the effect of chronic administration of dexamethasone (atrophy) could be due to the sum of the effects of repeated acute administrations causing the reprogramming of the activity of intracellular pathways controlling muscle mass [7].

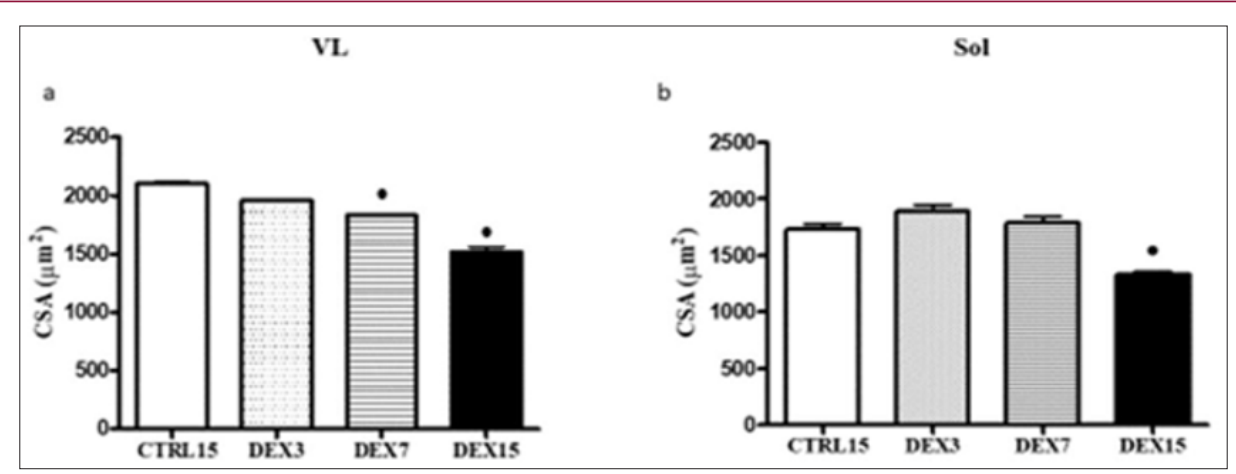

Figure 2: Cross section area (CSA) from cryosections of VL (a) and sol (b) of control mice (VL CTRL15 n=303; Sol CTRL15 n=314) after 3 days (VL DEX3 n=300; Sol DEX3 n=298), 7 days ( VL DEX7 n=312; Sol DEX7 n=300), and 15 days (VL DEX15 n=357; Sol DEX15 n= 322), of DEX treatment. CTRL15 mice open bar; DEX3mice, dotted bar; DEX7 mice, Striped bar; DEX15 mice, closed bar. Representative cryosections of VL (c) and Sol (d) for each experimental group. Data are re- plotted from caneapri et al. (2008) values are expressed as means \pm SD $\cdot$ P $<0.05$ vs CTRL15. 


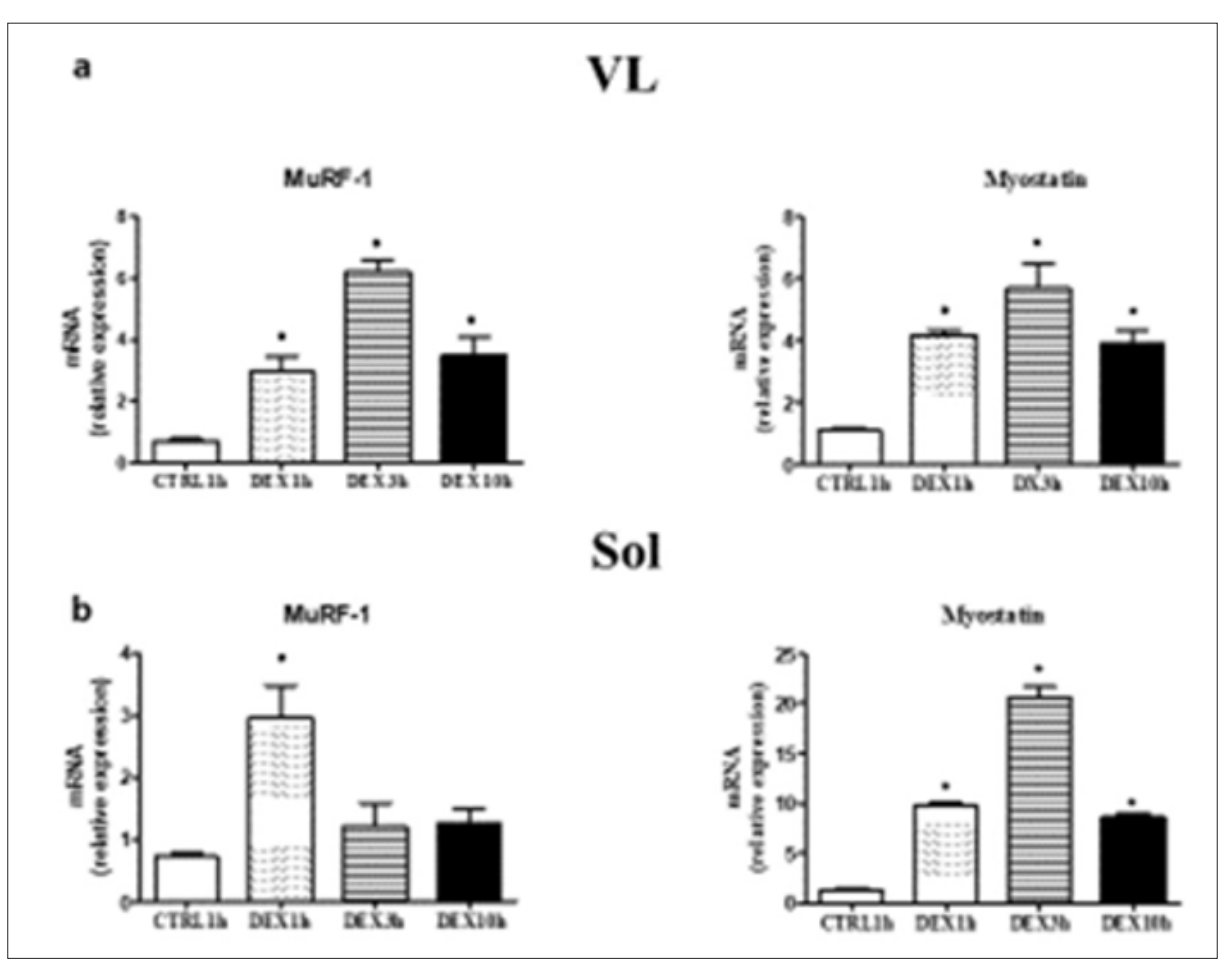

Figure3: MuRF-1 mRNA level in VL a) Sol (b) and myostatin mRNA levels in VL (c) and Sol (d) of control mice (CRLT15) and after 1 hour (DEX1h), 3 hours (DEX3h) and 10 hour (DEX10h) from a single DEX injection. CTRL1h mice (n=5), open DEX10h mice ( $\mathrm{n}=5)$ closed bar, Data are re-plotted from caneapri et al. (2008) Values are expressed as means \pm SD $\cdot \mathrm{P}<0.05$ vs CTRL1h.

\section{Conclusion}

Collectively the studies had shown that:

(i) 1 week of DEX treatment is able to cause muscle atrophy

(ii) Several responses occur very early (1 hour) following DEX single administration. From these observations follows the need to investigate the pathogenic mechanisms of steroid myopathy in the very early stages of treatment.

\section{Perspectives}

So far, no drug has proven successful in counteracting the development of steroid atrophy. More work is needed to text a potential therapy able to counteract the altered intracellular signalling pathways and atrophy following DEX treatment.

\section{References}

1. Minetto MA, Qaisar R, Agoni V, Motta G, Longa E, et al. (2015) Quantitative and qualitative adaptations of muscle fibers to glucocorticoids. Muscle Nerve.
2. Minetto MA, Lanfranco F, Motta G, Allasia S, Arvat E, et al. (2011) Steroid myopathy: some unresolved issues. J Endocrinol Invest 34(5): 370-375.

3. Schakman O, Kalista S, Barbé C, Loumaye A, Thissen JP (2013) Glucocorticoid-induced skeletal muscle atrophy. Int J Biochem Cell Biol, 45(10): 2163-2172.

4. Bottinelli R, Canepari M, Pellegrino MA, Reggiani C (1996) Force-velocity properties of human skeletal muscle fibres: Myosin heavy chain isoform and temperature dependence. Journal of Physiology-London, 495(2): 573-586.

5. Borina E, Pellegrino MA, D Antona G, Bottinelli R (2010) Myosin and actin content of human skeletal muscle fibers following 35 days bed rest. Scand J Med Sci Sports, 20(1): 65-73.

6. D Antona G, Pellegrino MA, Adami R, Rossi R, Carlizzi CN, et al. (2003) The effect of ageing and immobilization on structure and function of human skeletal muscle fibres. J Physiol 552(Pt 2): 499- 511.

7. Canepari M, Agoni V, Brocca L, Ghigo E, Gnesi M, et al. (2018) Structural and molecular adaptations to dexamethasone and unacylated ghrelin administration in skeletal muscle of the mice. J Physiol Pharmacol, 69(2). 
ISSN: 2574-1241

DOI: $10.26717 /$ BJSTR.2018.10.001889

Monica Canepari. Biomed J Sci \& Tech Res

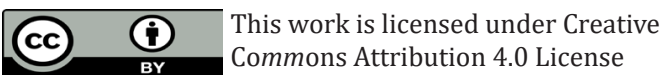

Submission Link: https://biomedres.us/submit-manuscript.php

$\begin{array}{ll}\text { BIOMEDICAL } & \text { Assets of Publishing with us } \\ \text { RESEARCHES } & \text { - Global archiving of articles } \\ \text { - Immediate, unrestricted online access } & \text { - Rigorous Peer Review Process } \\ & \text { - Authors Retain Copyrights }\end{array}$

